PAPER

\title{
Predictors of good outcome in medium to large spontaneous supratentorial intracerebral haemorrhages
}

\author{
M Castellanos, R Leira, J Tejada, A Gil-Peralta, A Dávalos, J Castillo, for the Stroke Project, \\ Cerebrovascular Diseases Group of the Spanish Neurological Society
}

J Neurol Neurosurg Psychiatry 2005;76:691-695. doi: 10.1136/jnnp.2004.044347

See end of article for authors' affiliations

.....................

Correspondence to: Dr José Castillo,

Department of Neurology, Hospital Clínico

Universitario Santiago de Compostela, Travesa da Choupana s/n, Santiago de Compostela 15706 ,

Spain; mecasti@usc.es

Received 27 April 2004

In revised form

16 July 2004

Accepted 19 August 2004
Objective: To determine potential predictors of good outcome in primary medium to large intracerebral haemorrhages $(\mathrm{ICH})$ which could be useful for selecting patients for surgical procedures.

Methods: Subjects were 138 patients with spontaneous hemispheric $\mathrm{ICH}>20 \mathrm{ml}$. They were nonsurgically treated and were admitted consecutively to 15 hospitals within the first 12 hours of symptom onset (mean (SD), 5.8 (3.1) hours). Haematoma volume was measured on computed tomography (CT) at admission. Stroke severity was assessed by the Canadian stroke scale (CSS). Good outcome was defined as modified Rankin score $\leqslant 2$ at three months.

Results: At the end of the follow up period, 45 patients (32.6\%) had good outcome. Baseline stroke severity, systolic and diastolic blood pressure, body temperature, and acute phase reaction biochemical markers (ESR, C-reactive protein, fibrinogen, neutrophil count) were significantly associated with good outcome in bivariate analyses. Of the initial CT scan variables, intraventricular contamination, deep location, mass effect, and greater $\mathrm{ICH}$ volume were related to poor outcome. On multiple logistic regression analysis, cortical location of bleeding (odds ratio 3.79 (95\% confidence interval 1.2 to 12.01 ); $p=0.023$ ), high CSS score (OR 2.3 (1.6 to 3.1); $<<0.0001$ ), and low fibrinogen concentrations (OR 0.92 $(0.87$ to 0.97$) ; p=0.001)$ were independent predictors of good outcome. These three factors correctly classified $85 \%$ of patients.

Conclusions: Good outcome in medium to large $\mathrm{ICH}$ can be predicted on admission by three readily assessable factors (CSS score, ICH location, and fibrinogen levels). These predictors may be helpful in selecting patients for surgical treatment.
$\mathrm{S}$ pontaneous intracerebral haemorrhage (ICH) accounts for approximately $4-14 \%$ of all strokes and is associated with a high mortality and morbidity. ${ }^{12}$ Between 32\% and $50 \%$ of patients die within the first month, ${ }^{34}$ and only $20 \%$ are independent six months after intracerebral bleeding. ${ }^{45}$ Despite the seriousness of the condition, the best therapeutic option for patients suffering from spontaneous supratentorial ICH remains to be established. However, on the basis of a few positive studies, ${ }^{6}{ }^{7}$ the Stroke Council of the American Heart Association has recommended the surgical removal of moderate to large lobar ICH in the case of young patients who are clinically deteriorating. ${ }^{8}$

Several clinical and radiological factors such as age, level of consciousness, hypertension, volume of the haematoma, volume of peripheral oedema, midline shift displacement on initial computed tomography (CT), and intraventricular spread of the bleeding appear to be markers of poor prognosis after spontaneous ICH. ${ }^{9-16}$ However, only a few studies have attempted to identify factors related to a favourable functional outcome in patients suffering from spontaneous supratentorial $\mathrm{ICH}^{11}{ }^{17}$ and most of these did not use haematological variables in their analyses.

Our aim in the present study was to investigate easily identifiable predictors of a good outcome in medium to large spontaneous supratentorial ICH, suitable for selecting patients who might benefit from surgical procedures while avoiding the harmful effects of surgery in those with potential for spontaneous recovery.

\section{METHODS}

We studied 138 patients (54\% male, mean (SD) age, 70 (11) years), consecutively admitted to 15 Spanish hospitals with a spontaneous supratentorial ICH of volume $>20 \mathrm{ml}$ diagnosed by cranial CT within the first 12 hours after symptom onset. The cut off value for ICH volume was in accordance with that used in clinical trials investigating the role of surgical evacuation in patients with spontaneous cerebral haematomas. ${ }^{18}$ The subjects were selected from a total of 266 patients recruited between May 1999 and April 2001 to a prospective multicentre study of spontaneous hemispheric ICH. The recruitment period for each hospital was different, resulting in more subjects being included by hospitals that recruited patients over a longer period of time (see appendix). Patients with haematoma secondary to head injury, congenital or acquired coagulation abnormalities, or any secondary cause of haemorrhage requiring surgical treatment such as cerebral aneurysm, arteriovenous malformation, or tumour were excluded.

The remaining 128 patients in the multicentre study were excluded for the following reasons: 114 had a haematoma volume $<20 \mathrm{ml} ; 10$ were lost to follow up at 90 days; and four were in a comatose state on admission, so would not have contributed to the objective of the study (the surgical removal of blood is of no benefit when there is a very low level of consciousness ${ }^{8}$ ).

The study was approved by the ethics committees of the different hospitals and informed consent was obtained from the patients or their relatives.

In summary, our protocol included a medical history recording potential vascular risk factors, clinical examination, blood and coagulation tests, 12-lead electrocardiogram, and chest radiography. All patients underwent cranial CT on admission. The scans were evaluated at the coordinating

Abbreviations: CSS, Canadian stroke scale; ICH, intracerebral haemorrhage; mRS, modified Rankin score 
centre by a single investigator who was blind to the clinical data. Topographic classification of the haematoma was assessed according to previously defined criteria. ${ }^{19}$ Haematomas that predominantly affected the subcortical white matter of the cerebral lobes were defined as lobar, while those in the basal ganglia, thalamus, or both were defined as deep. The presence of intraventricular blood associated with bleeding outside the ventricles was considered to represent intraventricular spread of the haematoma regardless of the total volume of intraventricular bleeding (which was not quantified). Neither the presence nor the quantity of subarachnoid haemorrhage was evaluated in this study. Mass effect was defined as the presence of midline shift displacement by $>5 \mathrm{~mm}$ on cranial CT. The presence of peripheral hypodensity surrounding the area of bleeding was also evaluated on the initial CT. The volume of hypodensity was calculated by subtracting the ICH volume from that of the total lesion, as previously described. ${ }^{19}$ The ICH volume was also calculated by using the formula $0.5 \times a \times b \times c$ (where $a$ and $b$ are the largest perpendicular diameters of the haemotoma measured on the CT scan, and $c$ is the number of slices multiplied by the slice thickness).
Stroke severity was evaluated by an experienced neurologist using the Canadian stroke scale $(\mathrm{CSS})^{20}$ on admission. The CSS measures level of consciousness, orientation, and weakness in arm, hand, and leg, with a total score ranging from 1.5 (maximum deficit) to 10 (absence of deficit). ${ }^{20}$ The modified Rankin score (mRS) was used to evaluate the outcome of patients at three months. Patients with an mRS of $\leqslant 2$ were considered to have a good outcome, while those with an mRS of $>2$ were considered to have a poor outcome.

After the initial evaluation, which was carried out in the emergency department, the patients were admitted into a neurological ward or acute stroke unit where they were attended by a specialised stroke and nursing team. Antihypertensive drugs were given if the systolic blood pressure was $>185 \mathrm{~mm} \mathrm{Hg}$ and/or the diastolic blood pressure was $>105 \mathrm{~mm} \mathrm{Hg}$. Metamizol or acetaminophen was used to treat hyperthermia (body temperature $>37.5^{\circ} \mathrm{C}$ ). Subcutaneous low dose heparin was given as prophylaxis against deep venous thrombosis and pulmonary thromboembolism. None of the patients received glucose solutions or steroids. Intravenous mannitol was given in 37 patients with suspected severe intracranial hypertension.

\begin{tabular}{|c|c|c|c|}
\hline Variable & Good outcome $(n=45)$ & Poor outcome ( $n=93$ ) & $\mathrm{p}$ Value \\
\hline Male sex & $18(40.0 \%)$ & $56(60.2 \%)$ & 0.030 \\
\hline Age (years) & $67.7(10.8)$ & $71.1(10.4)$ & 0.073 \\
\hline Mean time from stroke onset to admission (h) & $6.6(2.9)$ & $5.7(3.1)$ & $<0.001$ \\
\hline \multicolumn{4}{|l|}{ Vascular risk factors } \\
\hline Arterial hypertension & $26(57.8 \%)$ & $70(75.3 \%)$ & 0.048 \\
\hline Cigarette smoking & $18(40.0 \%)$ & $35(38.0 \%)$ & 0.853 \\
\hline Alcohol intake (>40 g/day) & $14(31.1 \%)$ & $24(25.8 \%)$ & 0.546 \\
\hline Diabetes mellitus & $9(20.0 \%)$ & $14(15.1 \%)$ & 0.473 \\
\hline Previous stroke or TIA & $8(17.8 \%)$ & $5(5.4 \%)$ & 0.156 \\
\hline \multicolumn{4}{|l|}{ Clinical characteristics on admission } \\
\hline Canadian stroke scale score & 5.7 (1.9) & $3.4(1.5)$ & $<0.001$ \\
\hline Vomiting & $11(25.6 \%)$ & $25(26.9 \%)$ & 1.0 \\
\hline Headache & $19(42.2 \%)$ & $15(16.1 \%)$ & 0.001 \\
\hline Seizures & $2(4.4 \%)$ & $7(7.5 \%)$ & 0.718 \\
\hline \multicolumn{4}{|l|}{ Radiological variables on cranial CT } \\
\hline Location of the ICH & & & 0.009 \\
\hline Deep & $26(57.8 \%)$ & 74 (79.6\%) & \\
\hline Cortical & $19(42.2 \%)$ & $19(20.4 \%)$ & \\
\hline Intraventricular bleeding & $11(24.4 \%)$ & $40(43.0 \%)$ & 0.039 \\
\hline Mass effect & $21(47.7 \%)$ & $68(73.1 \%)$ & 0.007 \\
\hline Peripheral hypodensity & $37(86.0 \%)$ & $89(95.7 \%)$ & 0.072 \\
\hline Volume of peripheral hypodensity (ml) & $14.9(18.0)$ & $18.4(17.3)$ & 0.278 \\
\hline Volume of the ICH (ml) & $58.2(26.4)$ & $78.2(54.2)$ & 0.004 \\
\hline Leukoaraiosis & $19(42.2 \%)$ & $43(46.2 \%)$ & 0.717 \\
\hline Old lesions & $8(17.8 \%)$ & $11(12.0 \%)$ & 0.431 \\
\hline \multicolumn{4}{|l|}{ Biochemistry and vital signs on admission } \\
\hline Plasma glucose $(\mathrm{mg} / \mathrm{dll})$ & $143.3(32.4)$ & $153.9(46.9)$ & 0.180 \\
\hline Packed cell volume (\%) & $41.2(3.7)$ & $42.3(4.3)$ & 0.172 \\
\hline Neutrophil count $\left(\times 10^{3} / \mathrm{mm}^{3}\right)$ & $8.3(2.9)$ & $11.0(7.3)$ & 0.018 \\
\hline Platelet count $\left(\times 10^{5} / \mathrm{mm}^{3}\right)$ & $192.3(61.1)$ & $198.4(62.3)$ & 0.589 \\
\hline Plasma fibrinogen* (mg/dl) & $359.8(87.6)$ & $461.0(144.2)$ & $<0.001$ \\
\hline Prothrombin time (\%) & $93.4(10.7)$ & $93.3(10.3)$ & 0.637 \\
\hline $\mathrm{aPTT}(\mathrm{s})$ & 29.8 (4.5) & $27.5(3.0)$ & 0.003 \\
\hline C reactive protein* $(\mathrm{mg} / \mathrm{l})$ & $3.7(5.1)$ & $13.2(13.4)$ & 0.020 \\
\hline $\mathrm{ESR}^{*}$ & $17.0(14.8)$ & $29.7(19.3)$ & $<0.001$ \\
\hline Systolic blood pressure $(\mathrm{mm} \mathrm{Hg})$ & $168.6(28.6)$ & $184.2(27.3)$ & 0.003 \\
\hline Diastolic blood pressure $(\mathrm{mm} \mathrm{Hg})$ & $93.5(17.3)$ & $100.9(17.9)$ & 0.021 \\
\hline Body temperature ( $\left.{ }^{\circ} \mathrm{C}\right)$ & $36.5(0.5)$ & $36.7(0.8)$ & 0.038 \\
\hline \multicolumn{4}{|c|}{$\begin{array}{l}\text { Values are mean (SD) or } n(\%) \text {. } \\
\text { *Fibrinogen, C reactive protein, and ESR levels were obtained from } 42,8 \text {, and } 35 \text { patients of the } 45 \text { with a good } \\
\text { outcome, and from } 87,16 \text {, and } 81 \text { patients of the } 93 \text { with a poor outcome. } \\
\text { aPT, activated partial thromboplastin time; CT, computed tomography; ESR, erythrocyte sedimentation rate; ICH, } \\
\text { intracerebral haemorrhage; TIA, transient ischaemic attack. }\end{array}$} \\
\hline
\end{tabular}




\section{Laboratory tests}

Blood samples were drawn from all patients on admission. Samples were collected in glass test tubes containing citrate and centrifuged at $3000 \times g$ for five minutes. Fibrinogen levels were analysed using a coagulometric method (PT-fibrinogen HS, Hemosil ${ }^{\mathrm{TM}}$ ) and $\mathrm{C}$ reactive protein using a cromogenic method (IL Test Protein C, Hemosil ${ }^{\mathrm{TM}}$ ). Biochemical variables and cell counts were obtained using standard methods. Determinations were carried out in an independent laboratory blinded to clinical and radiological data.

\section{Statistical analysis}

Comparisons of proportions between groups of patients were made using the $\chi^{2}$ test. Continuous variables are expressed as mean (SD), and compared using Student's $t$ test or the Mann-Whitney test as appropriate. We used the Spearman correlation to analyse the association between the clinical variables and biological acute phase reactants.

Logistic regression analysis was employed to find the best predictive model of good outcome. Age and those variables with a $\mathrm{p}$ value $<0.05$ in the univariate analyses were included as covariates. The positive correlation between the variables related to the acute phase response (biochemical or haematological markers and body temperature) precluded us from carrying out the analysis with all these variables in the same model. In the end, fibrinogen concentration was the variable used in the analysis as this gave the model with the greatest predictive capacity. Both forward and backward variable selection methods were used. We determined the sensitivity and specificity of the model and assessed goodness of fit with the Hosmer and Lemeshow statistic.

\section{RESULTS}

Of the 138 patients studied, $45(32.6 \%)$ had a good outcome at three months, $54(39.1 \%)$ were dependent (mRS 3-5), and $39(28.3 \%)$ had died. Table 1 summarises the main characteristics of the population studied. Patients with a good outcome were less likely to have a history of hypertension. The time from onset of the symptoms to admission was longer in patients with a good outcome, and they also more often had headache on admission. They had lower baseline stroke severity, lower systolic and diastolic blood pressure, and were more often male. The acute phase response was weaker in patients with a good outcome, as reflected by their lower baseline body temperature and lower fibrinogen concentrations, neutrophil count, erythrocyte sedimentation rates (ESR), and C reactive protein levels.

Among the radiological variables at baseline, larger volume of the ICH, deep location of the haematoma, intraventricular spread of bleeding, and mass effect were significantly related to poor outcome, whereas the volume of peripheral hypodensity did not differ between the two groups of patients.

In the logistic regression analysis, time from symptom onset to admission, headache, ICH volume, intraventricular bleeding, systolic blood pressure, and body temperature at

\begin{tabular}{|c|c|c|}
\hline Variable & Coefficient (SE) & OR $(95 \% \mathrm{Cl})$ \\
\hline $\begin{array}{l}\text { Constant } \\
\text { CSS at admission } \\
\text { Cortical ICH location } \\
\text { Fibrinogen levels } \\
\text { (by } 10 \mathrm{mg} / \mathrm{dl} \text { ) }\end{array}$ & $\begin{array}{r}-1.25(1.21) \\
0.81(0.16) \\
1.33(0.59) \\
-0.08(0.03)\end{array}$ & $\begin{array}{l}\overline{-} \\
2.26(1.63 \text { to } 3.13) \\
3.79(1.19 \text { to } 12.01) \\
0.92(0.87 \text { to } 0.97)\end{array}$ \\
\hline
\end{tabular}

$\mathrm{Cl}$, confidence interval; CSS, Canadian stroke scale score; $\mathrm{ICH}$, intracerebral haemorrhage; $O R$, odds ratio. admission were eliminated by both forward and backward stepwise variable selection. Inclusion of age, sex, history of hypertension, and mass effect provided no additional discriminative information. Table 2 shows the operational logistic regression model and odds ratios for each of the factors. The significant factors in the model were the CSS score $(p<0.0001)$, the cortical location of ICH $(p=0.023)$, and fibrinogen concentrations $(p=0.001)$. Patients with a cortical location of bleeding were almost four times more likely to be independent than patients with deep ICH, after adjustment for the other factors (sensitivity of the model for predicting a good outcome $=0.81$, specificity $=0.86$, overall probability of being correct $=0.85$ ). The Hosmer and Lemeshow statistic indicated that the fit of the model to the data was good $(\mathrm{p}=0.52)$.

\section{DISCUSSION}

Whereas medical treatment is recommended for patients with small cerebral haemorrhages, ${ }^{8}$ the best therapeutic option for those with medium to large spontaneous supratentorial haemorrhages remains controversial. Some studies have reported a lack of efficacy of surgical evacuation $^{21-24}$ whereas others have shown lower mortality in patients who underwent surgical removal of blood. ${ }^{6}$ Therapeutic strategies should be based on the prediction of the natural evolution of the ICH. Predictive models of mortality and morbidity, based on clinical and radiological variables, have already been developed in patients with spontaneous ICH. Mortality at 30 days is correctly predicted in $95 \%$ of patients by assessing the Glasgow coma scale (GCS) score, ICH volume, intraventricular spread of the bleeding, and pulse pressure..$^{12-14}$

Unlike previous studies that focused on mortality, we evaluated predictors of independence in the subgroup of patients with the worst prognosis. To the best of our knowledge, this is the first study seeking prognostic factors for good outcome in patients with large haematomas which are likely to be useful in evaluating the suitability of surgical procedures. A low severity of neurological deficit assessed by the CSS, together with a cortical location of the bleeding and low fibrinogen levels, accurately predicted a good outcome in $85 \%$ of our patients. The three variables selected by the model are easy to collect and clinically relevant. ${ }^{25}{ }^{26}$ In our opinion, these three factors include the most important elements related to ICH prognosis; the volume of the ICH had only a marginal effect because we were only studying large haematomas.

Dealing with these factors in turn, the CSS total score reflects the level of consciousness, the degree of limb paresis, and communication abnormalities, so it combines the verbal component of the GCS, arm strength, and the ability to walk, which have recently been reported and validated as good predictors of survival at 30 days and of survival in a nondisabled state at six months after ICH. ${ }^{11}{ }^{27}$

Second, the better outcome for lobar location probably reflects less motor disturbance and reduced vulnerability to the mass effect in cortical bleeding than in deep ICH when the volume exceeds $20 \mathrm{ml}$. A similar result was found in another report, ${ }^{28}$ but other studies have failed to demonstrate an association between location and outcome in the ICH overall. ${ }^{11} 1617$

Third, the plasma fibrinogen level in this study was the most powerful marker of the acute phase response to the brain injury, an effect that was shared by other biological markers of the stress reaction measured at admission, such as body temperature, neutrophil count, ESR, and C reactive protein. Fibrinogen levels have been associated with ischaemic stroke mortality and poor outcome, ${ }^{29-31}$ but few data are available in patients with ICH. ${ }^{32-35}$ Although increased levels of fibrinogen 
may only reflect the amount of damaged cerebral tissue, we did not find any relation between fibrinogen concentrations in the blood and the volume of the haematoma; however, a modest but significant correlation was found between fibrinogen levels and the volume of the peripheral hypodensity on admission (data not shown). We previously reported the association between high levels of inflammatory molecules within 24 hours of ICH onset and the volume of the perihaematoma brain oedema measured on days 3 to 4 after the stroke, which supports the view that oedema is an indicator of the inflammatory response induced by the ICH. ${ }^{19}$ As it has been shown that fibrinogen increases in response to inflammatory molecules, ${ }^{36}$ we hypothesise that fibrinogen levels may be reflecting the activation of inflammatory mechanisms responsible for tissue damage around the haematoma.

The duration of the follow up has been highly variable in previous ICH prognostic studies. Some investigators have evaluated outcome at the time of discharge, ${ }^{17}{ }^{37}$ whereas others recorded outcome variables at two weeks, ${ }^{10}$ one month, ${ }^{11}{ }^{14}$ three months, ${ }^{16}$ six months, ${ }^{11}$ or even one year. ${ }^{28}$ Though few patients with supratentorial ICH have a change in their outcome status beyond two weeks, some do continue to improve. ${ }^{10}$ The inclusion of these patients, otherwise most probably misclassified if the outcome evaluation is not delayed until the third month of evolution, probably resulted in a strengthening of our model.

Because this is a multicentre study, enrolled patients were admitted to different hospitals with different neurological wards or stroke units. As specialised medical and nursing personnel were taking care of the patients during the time they were in the hospital and the same protocols of treatment were followed in the different institutions, it is unlikely that the outcome would have varied depending on the facility. However, some factors such as the type of care received by the patients or caregiver training for these particular patients-which were not included in our analysis-might have influenced the outcome after discharge from hospital. Moreover, because of the referral patterns and prehospital or in-hospital deaths (in the emergency department), some patients may have been missed in the present analysis. Although most of these were probably comatose and therefore would not have been included in the analysis, some might not have been in coma and so this is a possible source of bias.

The ability to predict the outcome of patients with medium to large spontaneous supratentorial ICH may improve the management of these patients. Where a good prognosis is identified, patients could be spared the potential risk of surgical interventions, and more accurate information could be given to the patients and their relatives. Finally, a predictive model in these patients may be a powerful tool for use in the selection of patients for clinical trials, avoiding the enrolment of those who could obtain no benefit from the procedure under investigation. ${ }^{27}$

\section{Conclusions}

We have developed a simple and easy to use model for predicting a good outcome in patients with a medium to large spontaneous supratentorial ICH. The accuracy of our model needs to be assessed by external validation with an independent prospective sample of similar patients.

\section{ACKNOWLEDGEMENTS}

We thank Dr María García (Biostatistics Unit, Clinical Research Department, Hospital Universitario Doctor Josep Trueta, Girona, Spain) for her comments on the design and interpretation of the statistical analysis. Participating centres, investigators, and the numbers of patients studied are listed in the appendix. Partial results of this investigation were presented at the 27th International Stroke Conference in San Antonio, Texas, USA in February 2002.

\section{Authors' affiliations}

M Castellanos, A Dávalos, Department of Neurology, Hospital Doctor Josep Trueta, Girona, Spain

Rogelio Leira, José Castillo, Department of Neurology, Hospital Clínico Universitario de Santiago, Santiago de Compostela, Spain

J Tejada, Department of Neurology, Hospital Virgen Blanca, León, Spain A Gil-Peralta, Department of Neurology, Hospital Virgen del Rocio, Seville, Spain

Competing interests: none declared

\section{REFERENCES}

1 Bamford J, Sandercock P, Dennis M, et al. A prospective study of acute cerebrovascular disease in the community: the Oxfordshire Community Stroke Project, 1981-86. 2. Incidence, case fatality rates and overall outcome at one year of cerebral infarction, primary intracerebral and subarachnoid hemorrhage. J Neurol Neurosurg Psychiatry 1990;53:16-22.

2 Kase CS, Crowell RM. Prognosis and treatment of patients with intracerebral hemorrhage. In: Kase CS, Caplan LR, eds. Intracerebral hemorrhage. Boston: Butterworth-Heinemann, 1994:467-89.

3 Anderson CS, Chakera TM, Stewart-Wynne EG, et al. Spectrum of primary intracerebral haemorrhage in Perth, Western Australia, 1959-90: incidence and outcome. J Neurol Neurosurg Psychiatry 1994;57:936-40.

4 Counsell C, Boonyakarnkul S, Dennis M, et al. Primary intracerebral haemorrhage in the Oxfordshire community stroke project. 2. Prognosis. Cerebrovasc Dis 1995;5:26-34.

5 Broderick J. Intracerebral hemorrhage. In: Gorelick PB, Alter M, eds. Handbook of neuroepidemiology. New York: Marcel Dekker, 1994:141-67.

6 Auer L, Deinsberger W, Niederkorn K, et al. Endoscopic surgery versus medical treatment for spontaneous intracerebral hematoma: a randomized study. J Neurosurg 1989;70:530-35.

7 Zuccarello M, Brott T, Derex L, et al. Early surgical treatment for supratentorial intracerebral hemorrhage. A randomized feasibility study. Stroke 1999:30:1833-9.

8 Broderick JP, Adams HP, Barsan W, et al. Guidelines for the management of spontaneous intracerebral hemorrhage. A statement for healthcare professionals from a special writing group of the Stroke Council, American Heart Association. Stroke 1999:30:905-15.

9 Fieschi $C$, Carolei A, Fiorelli $M$, et al. Changing prognosis of primary intracerebral hemorrhage: results of a clinical and computed tomographic follow-up study of 104 patients. Stroke 1988;19:192-5.

10 Portenoy RK, Lipton RB, Berger AR, et al. Intracerebral haemorrhage: a model for the prediction of outcome. J Neurol Neurosurg Psychiatry 1987;50:976-9.

11 Daverat P, Castel JP, Dartigues JF, et al. Death and functional outcome after spontaneous intracerebral hemorrhage. A prospective study of 166 cases using multivariate analysis. Stroke 1991;22:1-6.

12 Tuhrim S, Dambrosia JM, Price TR, et al. Prediction of intracerebral hemorrhage survival. Ann Neurol 1988;24:258-63.

13 Tuhrim S, Dambrosia JM, Price TR, et al. Intracerebral hemorrhage: external validation and extension of a model for prediction of 30-day survival. Ann Neurol 1991;29:658-63.

14 Broderick JP, Brott TG, Duldner JE, et al. Volume of intracerebral hemorrhage. A powerful and easy-to-use predictor of 30-day mortality. Stroke 1993;24:987-93.

15 Qureshi Al, Safdar K, Weil J, et al. Predictors of early deterioration and mortality in black Americans with spontaneous intracerebral hemorrhage. Stroke 1995;26:1764-67.

16 Gebel JM, Jauch EC, Brott TG, et al. Relative edema volume is a predictor of outcome in patients with hyperacute spontaneous intracerebral hemorrhage. Stroke 2002;33:2636-41.

17 Lisk DR, Pasteur W, Rhoades $\mathrm{H}$, et al. Early presentation of hemispheric intracerebral hemorrhage: prediction of outcome and guidelines for treatment allocation. Neurology 1994;44:133-9.

18 Mendelow AD. Surgical trial in intracerebral haemorrhage (STICH). Acta Neurochir Suppl 2000;76:521-2.

19 Castillo J, Dávalos A, Álvarez-Sabín J, et al. Molecular signatures of brain injury after intracerebral hemorrhage. Neurology 2002;58:624-9.

20 Cote R, Battista RN, Wolfson C, et al. The Canadian neurological scale: validation and reliability assessment. Neurology 1989;39:638-43.

21 McKissock W, Richardson A, Taylor J. Primary intracerebral hemorrhage: a controlled trial of surgical and conservative treatment in 180 unselected cases. Lancet, 1961; ii, 222-6.

22 Juvela S, Heiskanen O, Poranen A, et al. The treatment of spontaneous intracerbral hemorrhage: a prospective randomized trial of surgical and conservative treatment. J Neurosurg 1989;70:755-8.

23 Batjer HH, Reisch JS, Allen BC, et al. Failure of surgery to improve outcome in hypertensive putaminal hemorrhage: a prospective randomized trial. Arch Neurol 1990;47:1103-6.

24 Morgenstern LB, Frankowski RF, Shedden P, et al. Surgical treatment for intracerebral hemorrhage (STICH): a single-center, randomized clinical trial. Neurology 1998;51:1359-63.

25 Kwakkel G, Wagenaar RC, Kollen BJ, et al. Predicting disability in stroke: a critical review of the literature. Age Ageing 1996;25:479-89.

26 Laupacis A, Sekar N, Stiell JG. Clinical prediction rules: a review and suggested modifications of methodological standards. JAMA 1997;277:488-94. 
27 Counsell C, Dennis M, McDowall M et al. Predicting outcome after acute and subacute stroke. Development and validation of new prognostic models. Stroke 2002;33:1041-7.

28 Juvela S. Risk factors for impaired outcome after spontaneous intracerebral hemorrhage. Arch Neurol 1995;52:1193-200.

29 Suárez C, Castillo J, Suárez $\mathrm{P}$, et al. Valor pronóstico de factores analíticos hemorreológicos en el ictus. Rev Neurol 1996;24:190-2.

30 Longo-Mbenza B, Tonduangu K, Muyeno K, et al. Predictors of strokeassociated mortality in Africans. Rev Epidemiol Sante Publique 2000:48:31-9.

31 Dávalos A, Castillo J, Pumar JM, et al. Body temperature and fibrinogen are related to early neurological deterioration in acute ischemic stroke. Cerebrovasc Dis 1997; 7:64-9.

32 Thavarai V, Behari M, Prasad K, et al. Haemorheological factors in the pathophysiology of acute stroke. Neurol Res 1992;14(suppl 2):92-3.

33 Montaner J, Álvarez-Sabín J, Abilleira S, et al. Reactantes de fase aguda en el ictus: diferencias entre el infarto isquémico y la hemorragia intracerebral. Med Clin (Barc) 2001;116:54-5.

34 Fujii Y, Takuchi S, Harada A, et al. Hemostatic activation in spontaneous intracerebral hemorrhage. Stroke 2001;32:883-90.

35 Lip GY, Blann AD, Faroogi IS, et al. Sequential alterations in haemorheology, endothelial dysfunction, platelet activation and thrombogenesis in relation to prognosis following acute stroke: the West Birmingham Stroke Project. Blood Coagul Fibrinolysis 2002;13:339-47.

36 Margaglione M, Grandone E, Mancini FP, et al. Genetic modulation of plasma fibrinogen concentrations: possible importance of inteleukin-6. J Thromb Thrombolysis 1996:3:51-6.

37 Flemming KD, Wijdicks EF, Li H. Can we predict poor outcome at presentation in patients with lobar hemorrhage. Cerebrovasc Dis 2001;3:183-9.
APPENDIX

PARTICIPATING CENTRES, INVESTIGATORS, AND (NUMBERS OF PATIENTS STUDIED)

Hospital Clínico Universitario, Santiago de Compostela: José Castillo, Rogelio Leira (62); Hospital Universitari Doctor Josep Trueta, Girona: Antonio Dávalos, Yolanda Silva (47); Hospital Virgen del Rocío, Sevilla: Alberto Gil Peralta, Enrique Montes (21); Hospital Virgen Blanca, León: Javier Tejada (21); Hospital Clinic, Barcelona: Ángel Chamorro, Nicolás Vila (20); Hospital Arquitecto Marcide, Ferrol: Francisco López, José Aldrey (19); Hospital Clínico, Valencia: José Láinez, Raquel Chamarro (17); Hospital Provincial, Pontevedra: Manuel Martínez-Seijo (16); Hospital de La Princesa, Madrid: José Vivancos, Raquel González (13); Hospital Clínico, Madrid: José Egido (9); Hospital Vall d'Hebrón, Barcelona: José Álvarez-Sabín, Joan Montaner (8); Hospital Gregorio Marañón, Madrid: Antonio Gil, Fernando Díaz (5); Hospital Virgen de la Concha, Zamora: José Gómez (3); Hospital La Paz, Madrid: Exuperio Diez-Tejedor (3); Hospital Clínico, Zaragoza: Enrique Mostacero (2). 